Voix et Images

volxetimages

\title{
TUER ENTRE NOUS
}

\section{Anatomie d'un coupable en terres littéraires québécoises}

KILLING AMONG OURSELVES

ANATOMY OF A GUILTY PARTY IN THE QUÉBEC LITERARY

REALM

\section{MATAR ENTRE NOSOTROS \\ ANATOMÍA DE UN CULPABLE EN TIERRAS LITERARIAS QUEBEQUENSES}

\section{David Bélanger et Cassie Bérard}

Volume 45, numéro 1 (133), automne 2019

La région dans la littérature du Québec

URI : https://id.erudit.org/iderudit/1068994ar

DOI : https://doi.org/10.7202/1068994ar

Aller au sommaire du numéro

\section{Éditeur(s)}

Université du Québec à Montréal

\section{ISSN}

0318-9201 (imprimé)

1705-933X (numérique)

Découvrir la revue

Citer cet article

Bélanger, D. \& Bérard, C. (2019). TUER ENTRE NOUS : anatomie d'un coupable en terres littéraires québécoises. Voix et Images, 45(1), 15-28.

https://doi.org/10.7202/1068994ar

\section{Résumé de l'article}

Cet article s'intéresse au « retour » que fait le régionalisme, celui idyllique du début du $\mathrm{xx}^{\mathrm{e}}$ siècle, dans la littérature québécoise récente. Ce retour, plus précisément, rend compte de crimes dans un univers sachant difficilement les contenir. Dans Le discours sur la tombe de l'idiot (2008) de Julie Mazzieri, Le chasseur inconnu (2014) de Jean-Michel Fortier et Trois fois la bête (2015) de Zhanie Roy, nous rencontrons effectivement des meurtres, sans que les infrastructures ne permettent de les traiter : ni enquêteur, ni médecin légiste, ni quelque instance judiciaire n'apparaissent dans ces fictions. Une telle représentation du crime permet de conceptualiser ces oeuvres comme des " actualisations à distance ", c'est-à-dire que le mouvement de retour s'avère sciemment problématisé selon les conceptions éthiques contemporaines. Par le biais des questions de l'origine et de l'autorité, comme interrogations au coeur de ces fictions critiques de l'idéal régionaliste, cet article entend éclairer ce geste de création important dans la production récente. 


\author{
T UER E NTRE NOUS \\ Anatomie d'un coupable \\ en terres littéraires québécoises
}

$+++$

DAVID BÉLANGER

Université McGill

CASSIE BÉRARD

Université du Québec à Montréal

Mais je suis pas détective. Je peux me tromper ${ }^{1}$.

Ce serait peut-être à un juge de [trancher], mais il n'y a pas de juge dans le village² .

Quelle bête a bien pu laisser ce genre de marque? Elle ne veut pas le savoir.

Ce n'est ni sa place ni son rôle ${ }^{3}$.

On sait que le crime n'a guère épargné les régions québécoises d'antan. En ce sens, l'idéal de paix qu'expriment nos romans idylliques ${ }^{4}$, n'acceptant pour seule adversité que l'autre anglais, constitue une construction largement promue par le régionalisme s'érigeant sur les mœurs pures et simples des Canadiens français ${ }^{5}$. Si nos habitants subissent une "émotion violente» à la vue d'un corps assassiné, n'ayant «jamais eu l'occasion d'aller se blaser même sur l'idée de la mort, dans nos théâtres ${ }^{6}$ ", selon la fameuse formule de Philippe Aubert de Gaspé fils, de même, on

1 Julie Mazzieri, Le discours sur la tombe de l'idiot, Paris, José Corti, 2008, coll. «Domaine français», p. 51. Désormais, les références à cet ouvrage seront indiquées par le sigle DT suivi du folio, et placées entre parenthèses dans le texte.

2 Jean-Michel Fortier, Le chasseur inconnu, Montréal, La Mèche, 2014, p. 17. Désormais, les références à cet ouvrage seront indiquées par le sigle $C I$ suivi du folio, et placées entre parenthèses dans le texte.

3 Zhanie Roy, Trois fois la bête, Montréal, À l'étage, coll. «Noir», 2015, p. 46. Désormais, les références à cet ouvrage seront indiquées par le sigle TF suivi du folio, et placées entre parenthèses dans le texte.

4 Cette formule se trouve au cœur du Roman sans aventure (Isabelle Daunais, Montréal, Boréal, 2015, 222 p.). Si les prémisses de Daunais demanderaient à être discutées, il appert qu'elles mettent en évidence des socles idéologiques entourant la production romanesque du xix siècle et du début du xx siècle. Pierre Hébert, dans un article sur «l'assassinart», évoque cette idylle idéologiquement appelée par les HenriRaymond Casgrain, Camille Roy et Lionel Groulx, «car, écrit-il, au pays de Québec, on peut mourir gelé dans la forêt, mais on ne tue pas ou, à tout le moins, on ne se représente pas comme tueur ». Pierre Hébert, «De "l'assassinart" : réflexion sur nos premiers meurtres littéraires (1835-1837)», Bernard Andrès et Marc André Bernier (dir.), Portrait des arts, des lettres et de l'éloquence au Québec (1760-1840), Québec, Presses de l'Université Laval, coll. «République des lettres. Symposiums», 2002, p. 399.

5 Nous empruntons ici l'expression de Patrice Lacombe s'excusant au terme de La terre paternelle (Montréal, Bibliothèque québécoise, coll. «Littérature. BQ», 1993 [1846], p. 80) de ne pas offrir un bain de sang.

6 Philippe Aubert de Gaspé (fils), L'influence d'un livre, présentation, chronologie et bibliographie de Claude Lamy, Montréal, Bibliothèque québécoise, coll. «Littérature. BQ», 1995 [1837], p. 52. 
en trouve pour exploiter sans remords les «idiots du village», les forçant à la bêche sous un soleil de plomb jusqu'à ce qu'ils meurent d'insolation. «I a ben crevé, le bougre», annoncera le frère exploiteur à sa femme chez Albert Laberge. "En v'là un bon débarras ${ }^{7}$ ", trouvera-t-elle à répondre. Certains crimes émeuvent davantage que d'autres...

Dans son étude sur l'imaginaire des crimes célèbres au Québec, Alex Gagnon avance que les criminels ne sont rendus dicibles, représentables, qu'au sein d'une «communauté du dehors [appartenant] à la communauté de ceux et celles qui, monstres, bêtes ou démons, n'en ont aucune autre». "Construisant l'espace de son ici », la société québécoise de la fin du XIXe siècle peine à accueillir les criminels, plus souvent rejetés: «Extérieurs de la civilisation [...]. Extérieurs au territoire de cet ici lorsqu'on les condamne [...]. Extérieurs, toujours, à l'ensemble de lois et de normes qui constitue le socle de la vie sociale ${ }^{8}$.» Ainsi, postule-t-on, tout un ensemble de crimes intérieurs, intégrés au socle de la vie sociale, restent indicibles dans l'imaginaire, et quasiment impensables. "I a ben crevé», résume l'habitant ayant tué son frère: ce crime est au-dessus des lois. Alors, la littérature contemporaine est là pour faire retour.

Notre article se donne pour objectif d'observer un déplacement de l'imaginaire régional dans le roman québécois contemporain. Il s'agit, pour le trio de romans de 2000 à aujourd'hui que nous étudierons, de représenter la région d'antan en laissant voir les failles de cette paix sociale, d'interroger ces «lois et normes» qui repoussent le criminel en dehors de la communauté.

Convenons toutefois que le retour à la région qu'esquissent les œuvres à l'étude ne s'accompagne d'aucune velléité archéologique, d'aucune ambition d'enquête révélant un en deçà de la ruralité d'autrefois. Nous avons droit plutôt à une actualisation à distance de ces villages d'antan. Pour reprendre l'heureuse formule de Jean-François Hamel, ces œuvres opèrent une politique de la lecture en ce qu'elles détournent «la philologie au profit de l'allégorie, passant de l'historisation des textes à leur actualisa-

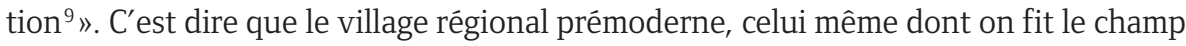
de bataille pour « axiomatiser le littéraire ${ }^{10}$ » au Québec, constitue un texte à réinvestir. Son détournement comme processus de mise à distance exprime certes l'ébranlement des croyances vis-à-vis d'un ordre classique et conventionnellement hiérarchique. Mais plus encore, l'actualisation qu'opèrent les romans que nous entendons analyser témoigne du déplacement d'un rapport à la vérité, que la modernité tâche d'expurger de toute croyance irrationnelle ou conventionnelle pour l'établir sur la base d'un processus de vérification scientifique, ou à tout le moins rationalisé, régi par un protocole spécialisé, allant des sciences juridiques aux sciences médico-légales, en passant par

7 Albert Laberge, La Scouine, Montréal, Typo, 1993 [1918], p. 106.

8 Alex Gagnon, La communauté du dehors. Imaginaire social et crimes célèbres au Québec (XIXe-XXe siècle), Montréal, Presses de l’Université de Montréal, coll. «Socius», 2016, p. 476-477.

9 Jean-François Hamel, Camarade Mallarmé. Une politique de la lecture, Paris, Éditions de Minuit, coll. «Paradoxe», 2014, p. 190

10 On retrouve cette proposition chez Lucie Robert, L'institution du littéraire au Québec, Québec, Presses de l'Université Laval, coll. «Vie des lettres québécoises/Centre de recherche en littérature québécoise», 1989, p. 193. 
la professionnalisation des enquêtes et des enquêteurs ${ }^{11}$. L'actualisation à distance constitue le geste littéraire permettant, à partir de l'époque contemporaine et avec son consensus axiologique, à la fois d'établir une valeur moderne de la vérité en montrant les lacunes des temps anciens et de présenter une modernité de la littérature, moins collaboratrice avec l'idyllique campagne que critique vis-à-vis de ses incartades. Invoquons, à titre d'exemple, le film de M. Night Shyamalan, The Village (2004), où la région ancienne est représentée en présence de l'époque contemporaine; elle apparaît ainsi au lecteur comme une parenthèse rendue compréhensible à l'aune du monde actuel. En effet, dans The Village, les protagonistes sont retirés dans un petit village, dans un apparent $\mathrm{XIX}^{\mathrm{e}}$ siècle. Isolée dans la clairière en raison de bêtes sanguinaires en sentinelles dans les bois - «Those we don't speak of -, la jeune génération de ce village ne peut que répondre aux traditions des Anciens, à leurs interdits et à leurs préceptes. Jusqu'à ce que les plus audacieux fomentent le plan d'aller quérir des «médicaments» à l'extérieur, et que le spectateur constate que très loin au-delà des bois serpentent des autoroutes, des villes: le monde normal du xxle siècle. Et que l'on comprenne dès lors que les sentinelles ne sont que des inventions des Anciens pour maintenir leur utopie passéiste.

Aucun des romans sur lesquels nous nous pencherons n'exhibe une telle structure ambiguë; celle-ci constitue pourtant une métaphore particulièrement révélatrice de la région d'antan représentée dans le roman québécois contemporain. L'époque, le plus souvent, est indéterminable. Les constructions sociales, à l'instar des bêtes inventées par les Anciens pour tenir captive la jeune génération dans le film de Shyamalan, s'avèrent saillantes; les autorités - souvent lourdement officielles, jamais professionnelles, ultimement morales et cléricales - sont dévoyées aussitôt présentées. Les meurtres constituent le produit de ces constructions sociales, et les bêtes qu'on ne sait nommer sont les boucs émissaires des dérèglements de l'ordre. Nous le verrons, dans les romans, cette région représentée suppose donc son cadre actantiel et sa résolution narrative.

Les trois romans cités en exergue de cet article seront donc explorés: Le discours sur la tombe de l'idiot de Julie Mazzieri, Trois fois la bête de Zhanie Roy et Le chasseur inconnu de Jean-Michel Fortier. On pourrait établir une productive généalogie entre ces œuvres et l'écriture de Gaétan Soucy, où l'isolement spatial se double d'un isolement intellectuel, notamment dans L'acquittement et dans La petite

11 Ce processus de professionnalisation des enquêtes s'avère tardif au Québec, comme on le sait. Il n'est pas anodin, à cet égard, qu'il se mette en branle à la faveur d'un crime teinté de politique. En effet, le meurtre de Blanche Garneau, en 1920, mettra au jour l'incurie des méthodes policières et du système juridique plus largement. Ainsi, on accusera le premier ministre et procureur général de la province, Louis-Alexandre Taschereau, de couvrir des fils de gens influents du Parti libéral du Québec. Les journaux Le Devoir et le Chronicle seront d'ailleurs poursuivis et accusés de diffamation; le journal The Axe ira encore plus loin, en accusant précisément certains individus. Au-delà du mystère que fut l'affaire Blanche Garneau, elle constituera le déclencheur de la Loi sur la police de 1922, qui donna naissance à la Sûreté provinciale et à un début de professionnalisation des enquêtes, avec notamment la création d'un «Service d'identité judiciaire", mené par les deux pionniers en Amérique du Nord de la médecine légale, Wilfrid Derome et Rosario Fontaine. Voir Les cahiers d'histoire de la Sûreté du Québec, vol. V, nº 1, mai 2015, en ligne: https:// www.sq.gouv.qc.ca/wp-content/uploads/2016/11/cahiers_histoire_vol5no1.pdf (page consultée le 9 août 2019). 
fille qui aimait trop les allumettes ${ }^{12}$; $\mathrm{l}^{\prime}$ ambition des romans que nous analyserons trouverait à se prolonger dans une production récente plantée dans un discours terroiriste nimbé de mythes et de légendes - ainsi de Borealium tremens de Mathieu Villeneuve ou encore de La bête creuse de Christophe Bernard ${ }^{13}$. En portant notre attention sur ces seuls trois titres, nous saurons en fait établir le fonctionnement de cette autorité sociale qui accuse et commet dans le même geste, pour ensuite approfondir cette question figurale par une question actoriale et énonciative: qui tue, et comment dire ces meurtres? Forcément, c'est l'autorité de la vérité dont discutent alors les œuvres.

\section{TUER L'ORIGINE}

«En plein jour»: voici la première phrase du Discours sur la tombe de l'idiot décrivant le meurtre de ce dernier. L'incipit déployé ne fait qu'en accentuer l'odieux: «En plein jour. Ils l'ont jeté dans un puits de l'autre côté du village. Ils l'ont pris par les jambes et l'ont fait basculer comme une poche de blé. En comptant un, deux, trois. Le maire et son adjoint.» $(D T, 9) C^{\prime}$ est aussi en plein jour que la petite Léa, première victime de la bête chez Zhanie Roy, trouve la mort; elle «avance avec confiance dans le blé qui lui arrive parfois jusqu'aux genoux [...], dans un paysage qu'elle connaît par cœur pour l'avoir parcouru toute sa vie» $(T F, 14)$. Et si le premier meurtre affligeant le village dans Le chasseur inconnu se commet le soir, lors d'une assemblée de routine, le public y assistant le met au jour: "La femme hausse les sourcils en nous voyant et jauge tout le monde du regard. [...] Au moment où elle montre [le Professeur] du doigt, il tire.» (CI, 38; nous soulignons)

Ce jour révèle le statut particulier des crimes. Peu clandestins, ils appartiennent à la vie du village, jamais ou presque confinés à la marge. Structurellement, ce trait se retrouve au cœur de la description de Mazzieri : en plein jour, phrase nominale, résonne avec le maire et son adjoint, les entrecroisant dans leur équivalence. Le meurtre devient officiel. Tuer l'idiot Midas participe d'une régulation sociale, dont doit se charger l'élu du village: «On a fait ce qu'on avait à faire. C'est fait.» $(D T, 37)$, arguera le maire pour calmer la culpabilité de son adjoint. Ce roman est d'ailleurs celui, parmi les trois, où la nécessité du crime se voit le mieux sémantisée, s'inscrivant dans une chaîne symbolique révélatrice.

En effet, le meurtre de l'idiot agit chez Mazzieri comme point de départ à toute une mécanique. Même si le cadavre du pauvre Midas ne sera jamais retrouvé au fond du puits où on l'a jeté, le spectre de sa disparition hantera les villageois de Chester au moment de la découverte d'un autre cadavre dans un fossé, celui d'une jeune femme sans origine. De même, la disparition de l'idiot propulsera le troisième et dernier meurtre, celui fermant le roman: Paul Barabé, ouvrier engagé venu de la

12 Gaétan Soucy, L'acquittement, Montréal, Boréal, 1997, 128 p.; Gaétan Soucy, La petite fille qui aimait trop les allumettes, Montréal, Boréal, 1998, 182 p.

13 Mathieu Villeneuve, Borealium tremens, Chicoutimi, La Peuplade, 2017, 366 p.; Christophe Bernard, La Bête creuse, Montréal, Quartanier, coll. «Polygraphe», 2017, 720 p. 
ville peu avant les assassinats, sera poignardé, devenant un véritable bouc émissaire du dérèglement affligeant la région. "En comptant un, deux, trois», dès l'incipit, le roman annonce l'enchaînement à venir. Il faut voir que le premier meurtre n'est pas seulement à l'origine de la trame romanesque; il constitue aussi bien le meurtre de l'origine, d'un début sans précédence, hors tradition donc, et hors continuité. Pour bien saisir cette origine, il faut se reporter à la fameuse distinction entre la nature et la culture. L'anthropologue Philippe Descola en trace productivement la généalogie dans son ouvrage phare Par-delà nature et culture. Par rapport à la nature, la culture, écrit-il, consiste en

[1]'idée que chaque peuple constitue une configuration unique et cohérente de traits matériels et intellectuels sanctionnés par la tradition, typique d'un certain mode de vie, enracinée dans les catégories singulières d'une langue et responsable de la spécificité des comportements individuels et collectifs et de ses membres, [...] idée [qui] émerge peu à peu [...] au tournant du $\mathrm{xx}^{\mathrm{e}}$ siècle $^{14}$.

La distinction s'établit, ainsi, au moment même où le régionalisme, au Québec, gagne en épaisseur idéologique et devient véritablement trait de culture. Cette culture, comme toute culture, a pour opposant constitutif, inscrit dans la dualité elle-même, l'objet de nature: cet objet échappe à la continuité de la tradition, brise la cohérence de la configuration socioculturelle. En ce sens, l'objet de nature est ce qui révèle l'avant-culture, l'être-au-monde sauvage, déréglé, et que la culture a pour quête sinon d'éliminer, du moins de domestiquer. L'idiot Midas, on le verra, ne sait être domestiqué. Si on dit de lui qu'il est un être contre nature, notamment issu d'une relation potentiellement incestueuse, ce qu'on veut vraiment exprimer alors tient plutôt à son opposition à la culture.

Deux traits de l'idiot justifient, pour le maire, de commettre le crime: une présence et une absence. En analepse, tentant de décrire son dégoût pour sa future victime, le maire parle d'une "parcelle de peau si blanche qu'il en avait eu la nausée. Mince comme de la soie et distendue par la graisse, avait-il dit. Là, en plein soleil, sans poils ni duvet, recouvrant le corps de cet homme; de ce ver» $(D T, 11)$. Cette blancheur s'insinuant entre gilet et pantalon laisse toutefois apparaitre une image pire, véritablement inacceptable: «Au milieu de cette panse veinée, il ne l'avait pas vu. L'ombilic. Il ne l'avait pas vu car il n'y en avait tout simplement pas.» $(D T, 11)$ «Mon Dieu, ce ventre sans nombril» $(D T, 12)$, répétera-t-on après que l'anecdote de la rencontre du maire et de l'idiot a été narrée; le maire «avait alors su qu'il fallait se débarrasser de cet idiot» $(D T, 12)$. Cette première justification peine à expliquer le geste décisif, aussi s'en adjoint-elle une autre, guère plus convaincante. La mère de Midas, madame Henri, est au désespoir depuis la disparition de son fils. Elle défend le cœur bon de l'idiot, demandant au maire si son idiot a continué à uriner sur la porte de la mairie après qu'elle l'a puni, montrant en cela qu'elle s'occupait de ses exactions, tentait de gérer son fils - et révélant au lecteur, du même coup, une faute commise. Alors, le maire se remémore l'horreur du geste, l'odeur d'ammoniac,

14 Philippe Descola, Par-delà nature et culture, Paris, Gallimard, coll. «Folio. Essais», 2015 [2005], p. 139-140. 
et jusqu'à la vision qui l'atteignit le plus sûrement: «Le maire avait été choqué: il s'attendait à un sexe d'enfant.» $(D T, 122)$

Avec le nombril absent et le sexe mature, nous avons deux signes qui affirment la monstruosité de Midas l'idiot - on l'associera à un ver - et le désignent comme victime. Sans l'origine dont le nombril témoigne, son sexe montre cependant un homme capable de se reproduire, équation inacceptable ${ }^{15}$. Sa situation familiale pointe en ce sens : l'idiot est sans père donc sans raison, comprend-on de la rumeur publique que livre le roman. De fait, la mère de Midas aurait eu maille à partir avec la police, raconte-t-on, après avoir dénoncé son frère qui l'aurait battue «quand il avait su qu'elle était enceinte» $(D T, 56)$ alors qu'elle n'était pas mariée. Le frère aurait alors été mis en prison, et elle, rejetée de la famille. La chaîne événementielle paraît encore plus lisible si on la déplie: femme enceinte, violence du frère, frère en prison racontent ainsi le crime des crimes, le tabou incestueux, comblant le manque du père. «On vous a jamais dit de pas faire ça avec les cousins?» ironise une femme du village, pointant cette piste que l'œuvre se garde de suivre.

Le deuxième meurtre s'appuie, sémiotiquement, sur le premier. Dans sa description, d'abord: «si blanches dans l'herbe mouillée, à peine repliées, si calmes, les jambes» (DT, 96), lit-on. Cette blancheur prêtée à Midas dans un premier temps ne caractérise la deuxième victime qu'après un imbroglio qui, effectivement, confondait ce corps et celui de l'idiot: "Mais laissez-la donc voir son Midas une dernière fois si c'est juste ça qu'elle veut. C'est son fils après tout!» lance la servante du curé à l'endroit de l'enquêteur, sur les lieux du crime. «Son fils? avait répété l'enquêteur [...]. C'est parce que ce que j'ai ici, ma petite dame, c'est une fille.» $(D T, 71)$ Enfin, l'appartenance familiale de cette jeune fille pose divers problèmes que s'empressera d'esquiver le maire dans une allocution à la municipalité, lui inventant un nom, pour le coup ${ }^{16}$. Il s'en enthousiasme auprès de son adjoint: "On ne pouvait pas mieux tomber: une traînée! Pas de famille, pas d'enfants, pas de mari. Une fille comme ça, ça meurt (il avait claqué les doigts), on vous la ramasse et plus personne n'en entend parler.» $(D T, 74)$ Ce personnage, qui ne trouve son origine que dans sa mort, rejoint donc le statut de Midas, sans précédence, pure discontinuité.

Le meurtre de l'origine - répété dans le roman - expose un manque de justification, nous l'avons vu: la colère du maire à l'endroit de l'idiot paraît sans fondement. Les commères du village en conviennent, elles aussi, après avoir étalé les circonstances de sa naissance: «De toute façon, même si on savait qui est le père, ça ne nous dirait toujours pas où est l'idiot ni pourquoi il a disparu.» (DT, 57; l'auteure souligne) L'origine manque, tout comme la destination, mais aucune logique ne permet de lier ces deux manques, de les inscrire dans une suite causale significative. De même, le roman de Mazzieri souligne l'indifférence dans laquelle se fond la mort de la jeune fille. Aucune forme d'enquête ne s'organise dans le village, le sujet perd doucement de sa pertinence dans les conversations. Ne reste qu'une haine envers

15 On a souvent rapproché l'écriture de Julie Mazzieri de celle de William Faulkner; si on pense d'abord, en raison de la trame policière, au roman Sanctuaire, ce «sexe de l'idiot» ne manque pas de référer à la castration de Benjy, autre «idiot», dans Le bruit et la fureur.

16 On ne peut que noter le statut «littéral» de ce nom: Isabelle Desmarais. Retrouvée dans un fossé inondé, elle, la «belle des marais». 
l'étranger, Paul Barabé, comme un soupçon, pour se souvenir du crime. Un seul personnage, à vrai dire, consacre certains soins à la jeune victime retrouvée dans le fossé; il s'agit du berger Simeoni, qui a découvert la dépouille avant qu'elle ne soit trouvée officiellement: "Je l'ai regardée bien longtemps pour qu'elle me raconte sa vie. Et j'ai imaginé qu'elle allait danser le vendredi soir avec ses amies. Qu'elle vivait dans un minuscule appartement.» $(D T, 135)$ Cette enquête inductive permet au berger de la baptiser, lui aussi: «J'ai eu la certitude qu'elle devait porter le nom d'une sainte et j'ai dit Eulalie, tout bas, pour la rassurer.» $(D T, 135)$ Vierge martyre, sainte Eulalie est surtout connue pour la cantilène qui lui est consacrée, grevée par un discours des origines. En effet, cette cantilène est le premier texte littéraire en français, et traite de son "corps beau, de son âme plus belle encore», rappelant que «Les ennemis de Dieu voulurent la vaincre/Et lui faire servir le Diable». Simeoni pointe ainsi un coupable, un diable, une force pure dans sa méchanceté accrochée à un monde férocement binaire - un diable qui se passe de motif raisonnable, d'ordre et de justification. Essayons maintenant de voir ce que cette structure du meurtre de l'origine nous enseigne sur l'ordre régnant dans ces régions.

Chez Jean-Michel Fortier comme chez Zhanie Roy, on constate l'opacité générale entourant les motifs du crime. Les meurtres surviennent; les assassins tentent de les justifier dans l'après-coup, sans succès. La trame de Trois fois la bête, en raison de son appartenance générique au polar, s'avère aisée à rendre: dans le petit village de Sainte-Clarisse, en 1935, surviennent trois meurtres d'enfants, tous éventrés, le foie disparu. Il faut le troisième meurtre, d'ailleurs, pour qu'on soupçonne qu'aucun loup, qu'aucune bête, n'aurait agi ainsi, aussi méthodiquement. Les regards se portent alors sur les villageois, sans que la rumeur s'en convainque. On organise plutôt une chasse pour tuer un loup; le succès de cette chasse met fin à la série de meurtres. Or, évidemment, un meurtrier agissait. Il se confesse, dans les dernières pages du roman, au curé, lequel est lié par son devoir et ne pourra le dénoncer. Le meurtrier est l'homme le plus influent du village, Aurel Labonté, aspirant maire, riche parvenu, revenant des États-Unis au volant de ce qui sera l'unique automobile de toute la région. Au "pourquoi» de son geste criminel, Aurel ne sait trop répondre: «J'y ai pas vraiment réfléchi.» $(T F, 209)$ Mais il spécifie bientôt, lui qui grandit fils sans père: «Je hais ces petits morveux, ces fils à papa. [...] Vous pouvez pas comprendre. [...] Le vide. Le trou en moi que je ne peux pas combler et que ces enfants remplissent.» (TF, 209) Dans cette explication pulsionnelle se joue l'origine qui manque, car c'est le comble originel des enfants qu'attaque le meurtrier. Ce discours psychologisant trouve un écho bien particulier dans le contexte communautaire où il s'exprime: dans une région lourdement érigée sur les continuités biologiques et institutionnelles, la discontinuité que représente Aurel - parti et revenu, sans père, originaire de la ville, élevé au village - justifie son crime. En avalant le foie de ses victimes, de même, il retrouve le chemin continu de son appartenance au village.

On constate une organisation similaire dans Le chasseur inconnu; en revanche, la dynamique conflictuelle du village compte beaucoup sur la structure ironique du roman. Les lundis soir, apprend-on d'entrée de jeu, les habitants du village se réunissent pour leur assemblée hebdomadaire officielle, dans la salle paroissiale sous 
l'église. Dirigée par le maire, hantée par l'autorité du curé, cette rencontre met en scène le personnel principal de l'endroit: le boulanger, la vieille fleuriste, la femme de l'apothicaire, le vénérable médecin, etc. Les comptes rendus de ces assemblées revêtent une étrangeté originale: ils sont narrés au nous, par un collectif mystérieux d'habitants. D'autres rencontres, secrètes celles-là - bien que le secret paraisse partagé par la quasi-totalité du village - , se déroulent les vendredis soir, dans la même salle paroissiale. Les participants de ces rencontres dirigées par le Professeur ne sont jamais nommés, bien que parfois reconnaissables. L'anonymat s'exprime jusque dans la narration, qui est encore une fois portée par un collectif, mais ici au on. C'est lors d'une rencontre du vendredi soir que le Professeur assassinera la coiffeuse Campeau d'un coup de fusil. Non initiée à ces réunions, elle les découvre par mégarde et se retrouve victime de son indiscrétion: "Qu'est-ce qu'elle m'a fait faire, celle-là? Vous l'avez vue, avec sa curiosité, ce qu'elle m'a fait faire, ah!» $(C I, 39)$ Cette rhétorique qui consiste à blâmer la victime doit être lue par-delà le réflexe de défense. En effet, elle invite à lire le crime à la lumière de l'organisation stricte du village, avec ses rencontres les lundis et ses rencontres les vendredis. Le respect des frontières étanches entre ces rencontres est garant de l'ordre et de la loi, et c'est cette frontière qu'a transgressée la victime. Qui plus est, cette transgression est ellemême justifiée par autre chose: la coiffeuse Campeau survient dans la salle paroissiale parce qu'elle est à la recherche de l'un de ses trois enfants, pour une énième fois égaré dans le village. On apprenait dès le premier chapitre que l'âge requis pour assister aux assemblées officielles du lundi avait été fixé à 18 ans, "sinon madame Campeau y amènerait ses enfants» $(C I, 13)$. Dans ce chapitre, il est également question du suicide récent de l'ancien maire, au comble d'un imbroglio gênant avec le maire actuel. L'ancien maire aurait accusé à tort Armelle Moche, la fille de celui qui deviendrait maire, de lui voler des crayons, appelant à l'enfermer au Poste, une bâtisse de béton sans fenêtres ayant tout à voir avec des oubliettes. Pour le dire simplement, dans Le chasseur inconnu, le problème vient des enfants. D'ailleurs, le deuxième assassinat sera celui d'Armelle Moche, sous la forme d'un homicide involontaire: afin de détourner l'attention du village, trop portée sur le meurtre de madame Campeau, le Professeur aurait ordonné au médecin de blesser la jeune fille lors de l'extraction de ses dents de sagesse, et d'ainsi provoquer une infection qui aurait emballé la rumeur. Comble de malchance, la jeune Moche ne s'en remet pas et meurt. Ajoutons que les enfants Campeau et Armelle Moche sont les seuls personnages que le roman identifie par leur enfance.

L'enfance, dans ce lieu reculé, sans liens ou presque avec le monde extérieur, signifie la continuité. Celle de la tradition, de même que la continuité biologique, exprimable en termes démographiques. Or, à la fin du roman, un Recenseur survient; il veut, pour le compte du gouvernement, établir la démographie précise du village. Tous les habitants s'y refusent. Vu son insistance, on se débarrasse de lui: on l'enferme dans le Poste, en lui mettant à tort le meurtre de madame Campeau sur le dos. Deus ex machina: lorsque rien ne va plus au village arrive le bouc émissaire, l'étranger doublement coupable. Il est coupable en raison de son altérité, puis de son désir de sonder la continuité démographique, celle même qui pose problème dans un univers férocement endogamique. 
La structure anthropologique que proposent ces lectures est claire: la région actualisée à distance se représente avec, en son cœur, le meurtre de l'origine, c'està-dire tout à la fois le meurtre de ce qui échappe à la domestication culturelle - les enfants, les sans-paroles - , et de ce qui en signifie l'avenir. On y tue ou y problématise ce qui continue la tradition - l'enfant, la mère - et on reporte la faute sur ce qui, de facto, est extérieur à cette tradition: la bête étrangère. Étienne Beaulieu, dans son article sur Le discours sur la tombe de l'idiot, note que "le passé du roman québécois hante ce récit comme un passé qui ne passe pas, comme une vie laissée à elle-même dans un arrière-pays que l'on avait oublié et qui revient sous la forme cauchemardesque ${ }^{17}$ ». Ce cauchemar est celui d'un ordre indifférent à la raison, aux causalités raisonnables, où dominent une autorité traditionnelle sans fondement logique, une rumeur sans organisation médiatique. Le crime, dès lors, devient un récit qu'on se raconte sans altérer la vie au quotidien, la spécificité des comportements individuels et collectifs d'une culture dont parle Descola. Le meurtre, comme fiction du réel, paraît inadmissible et il est dessiné dans les romans avec la force de son inadmissibilité, c'est-à-dire avec des contours qui confinent les personnages à l'aveuglement, à un espace, à une époque, en décalage.

\section{TUER TOUS ENSEMBLE}

La fameuse "perte des grands récits» que théorise Jean-François Lyotard participe d'un lieu commun qui a entraîné diverses actualisations postmodernes - fin de l'histoire, fin de la littérature, etc. Le geste que posent les romans de notre corpus consiste, dans une distance temporelle confuse ${ }^{18}$, à raconter depuis cette fin une époque ou un lieu où elle ne serait pas advenue. L'un des traits de cette perte absente se constate dans la distribution des responsabilités. Si la modernité est marquée par une constante rationalisation, comme l'écrit Max Weber, ou, selon Jürgen Habermas, par une différenciation des sphères de savoir et de valeur, menant à une spécialisation puis à une professionnalisation des sujets, tout ce processus se serait dévoyé et aurait donné naissance aux constats postmodernes: les sphères différenciées n'auraient plus su entrer en dialogue, le solipsisme et l'atomisation auraient nui à

17 Étienne Beaulieu, «La rumeur du passé. Le discours sur la tombe de l'idiot de Julie Mazzieri», Gilles Dupuis et Klaus-Dieter Ertler (dir.), À la carte. Le roman québécois (2005-2010), Francfort, Peter Lang, 2011, p. 270.

18 Étienne Beaulieu suppose que le roman de Mazzieri se déroule à l'époque contemporaine; en effet, sont mentionnés des autoroutes, des avions, le fait que tout le monde se déplace en automobile et utilise le téléphone. Toutefois, aucun appareil électronique n'est nommé, pas même la télévision (on évoque la radio). Marceau, de la laiterie, apporte une bouteille de lait à la porte de ses clients tous les jours; les personnages sont assez unanimement attachés à l'église, et le curé constitue «l'autorité suprême». D’ailleurs, si on paye en «dollars» et on arbore un phrasé souvent québécois, tel que le remarque Beaulieu, on évoque parfois les «communes» voisines, faisant pénétrer une dénomination proprement française (ibid., p. 267-275). L'indécision est structurelle dans le roman de Mazzieri, comme dans Le chasseur inconnu. Dans ce dernier, outre le téléphone, aucun objet technologique n'apparait. Par contre, on évoque une guerre passée et une guerre présente, ce qui laisse imaginer les années 1940. Le roman de Zhanie Roy seul est bien situé (en 1935). 
la lisibilité générale du monde, l'éclatement des unions narratives traditionnelles et modernes - religieuses, marxistes, mythiques - aurait fait perdre tout récit aux communautés ${ }^{19}$.

Dans les romans qui nous intéressent, aucun certifié professionnel ou presque: plus sûrement, des rôles typiques sont distribués - vicaire, maire, notaire, sacristain, ferblantier, berger, curé - qui rendent fonctionnel le village, et les seuls professionnels frais émigrés de la ville ne se mêlent pas à la vie du village ni à la trame de l'intrigue. Ils sont fonctionnaire et traductrice dans Le discours sur la tombe de l'idiot ${ }^{20}$, par exemple. Diverses extensions font de l'attribution des tâches une affaire quelque peu arbitraire: celui qui occupe le rôle de médecin agit également comme dentiste chez Fortier, le bedeau occupe les charges de fossoyeur, ou la sagefemme, chez Roy, celles d'embaumeur, liant les professions de la vie et de la mort. La première conséquence de cette indifférenciation des sphères de valeur se constate toutefois dans l'autorité de certains sujets, et par voie de conséquence, dans l'autorité de certains récits portant le crime. Voilà ce que révèlent les exergues en tête de cet article: c'est parce qu'il n'existe aucun professionnel du crime - détective, juge, médecin légiste - que sa narration est laissée à ceux qui peuvent en tirer avantage.

On a vu, chez Mazzieri, que le meurtre de l'idiot enrôlait les deux autres; de même, dans Le chasseur inconnu, Armelle Moche n'est assassinée que pour faire taire les rumeurs sur la mort de madame Campeau. Le troisième meurtre, quant à lui, consistant à jeter le Recenseur au fond d'un puits - ici, dans des oubliettes permet, à l'instar du meurtre de Paul Barabé chez Mazzieri, de mettre fin aux crimes en leur assurant une signification. Cette structure de causalité se fait encore plus révélatrice dans Trois fois la bête.

Après le meurtre de la petite Léa, le village de Sainte-Clarisse voit s'affronter deux puissances. D'un côté, le curé Damase Couture, condamné par un cancer, veut offrir un legs au village où il a œuvré durant nombre d'années; de l'autre, Aurel Labonté, homme matérialiste, riche et pragmatique, veut moins léguer quoi que ce soit que faire avancer sa communauté. La mort de Léa constitue l'enjeu qui les oppose: le legs du curé prend la forme d'un nouveau cimetière - «Ce sera le cimetière Damase-Couture...» $(T F, 58)$ - , qu'il situe près de la rivière, dans une zone qui, comme le criera Aurel en pleine messe, s'inonde chaque printemps. Devant cet outrage fait à la mémoire des défunts, Aurel s'élève et offre un terrain qui lui appartient, plus apte à accueillir le repos éternel. Sur ce terrain, précisément, la querelle se cristallise. En fait, l'opposition prend diverses formes. Afin de faire valoir la préséance de leur autorité, les deux sujets tâcheront d'abord de se faire les porte-parole du village: qui, des deux, exprime le mieux les intérêts de la population? «Ne prenez pas la parole au nom des villageois, quand vous êtes le seul à vous opposer réellement à mon idée» $(T F, 60)$, arguera le curé. Cette prise de parole collective concerne, au premier chef, l'appartenance à cette communauté,

19 Sur ces questions, voir l'ouvrage synthèse de Perry Anderson, Les origines de la postmodernité, traduit de l'anglais par Natacha Filippi et Nicolas Vieillescazes, Paris, Prairies ordinaires, coll. «Penser/Croiser», 2010 [1998], $185 \mathrm{p}$.

20 Étienne Beaulieu suggère qu'il s'agit d'une autoreprésentation de l'auteure qui «se positionne dans son propre roman en caméo, empruntant quelques-uns des traits des Pearson» («La rumeur du passé», p. 269). 
son inscription dans sa continuité. Le curé remettra en doute le lien d'Aurel avec les siens, et ce dernier rétorquera: "Vous osez me dire que je suis pas d'ici? Vous, qui vivez dans le village depuis des années et qu'on traite encore d'étranger, vous osez me dire que je suis pas d'ici.» (TF, 62) L'intégration dans le milieu devient le gage de l'autorité et, par conséquent, comme le souligne une scène importante peu avant la découverte du troisième cadavre, l'étrangeté génère du soupçon. En effet, alors que le curé a réuni dans le presbytère les principaux notables du village pour faire la lumière sur les crimes, il s'en prend ouvertement à Aurel, l'accusant. Ce dernier se défendra: «Pour votre projet de cimetière, ces morts tombent à point, si vous me permettez l'expression. De petits êtres purs pour honorer votre Seigneur, n'est-ce pas exactement ce que vous souhaitez, monsieur le curé?» $(T F, 147)$ Le meurtre, dans ce monde incapable de le penser rationnellement, devient un sème pour (r)établir un ordre. Celui immémorial et spirituel, qui assure la mémoire du curé, et celui pragmatique et matérialiste, qui devrait assurer la prise de pouvoir de l'aspirant maire, Aurel. Le meurtre devient le nœud d'une confrontation entre divers récits, de même: l'un avec Dieu pour guide, punissant et pardonnant; l'autre, plus confus et suspect, comme indicible, rattaché au souvenir amer, au secret, puis à la recherche d'une impureté pour tacher l'idylle de la campagne. C'est pourquoi un notable, devant la querelle sans issue de Damase et Aurel, présentera l'évidence que nul ne voudra remettre en doute, vérité poppérienne: «Jusqu'à preuve du contraire, rien ne contredit l'hypothèse de la bête sauvage. Pourrions-nous avoir affaire à un loup enragé ?» $(T F, 148)$ À la fin, le loup enragé, la bête bouc émissaire, est tué. Le curé, sur son propre lit de mort, suffoque devant la confidence d'Aurel Labonté. «Les gens ne croiront pas à cette histoire de loup», dira le curé, ce à quoi l'assassin opposera, soulignant tout l'enjeu de la lutte narrative dont il sort vainqueur: «Ils y croient déjà. Le cadavre de la bête est dans ma voiture. Les morts arrêteront avec sa disparition.» $(T F, 211)$

Le roman de Zhanie Roy fait cependant bien davantage que de souligner la victoire d'un récit. Dès le commencement, la narration distribue les soupçons, conformément à la forme policière. La fille du bedeau, Blanche, 22 ans, prête à se marier, est «sauvageonne, indomptée» (TF, 21). C'est elle qui découvre le premier cadavre, la petite Léa; aussi la soupçonne-t-on lors de l'assemblée d'enquête, au grand désarroi de son père. La mère de Blanche, Rose-Délima, enceinte, est aussi torturée par la culpabilité: des visions lui montrant les petits cadavres avant le crime, sortes de prémonitions, l'amènent à se soupçonner elle-même. Lors de la chasse au loup, dans une narration intercalée, Rose-Délima accouche, en proie à des délires. Elle veut avouer son crime: «Tu as rien fait, Rose-Délima. C'est pas toi» (TF, 195), oppose la sagefemme. Elle meurt en couches, au moment même où le loup trépasse. Au petit matin, Aurel part avec la dépouille de la bête, et Blanche l'indomptée le supplie de l'amener avec lui en ville, où la vie paraît moins contrainte qu'au village. Ainsi, tous ceux qui auront été objets de soupçons - loup, femmes, aspirant maire et curé - trépassent ou partent ce jour-là. Les crimes arrêtent par la suite, et la vie reprend; il faut la confidence d'Aurel Labonté dans ce monde sans autorité pour intriquer les causes et les conséquences. Mais ensuite les traces s'effacent, comme pour assurer au village son ordre inébranlable. 
On retrouve ici, en deçà de l'opposition entre la nature et la culture, celle entre le muthos et le logos; si chez les peuples anciens le récit conserve des «fonctions épistémologiques importantes", le développement de la science, et plus encore de la science positiviste, récuse quasiment toute valeur de savoir aux narrations, leur préférant les notions de «structure, de construction, de transformation ${ }^{21}$ ». En l'absence de savoirs rationnels, raconte Zhanie Roy dans son actualisation de la région d'antan, l'autorité narrative peut tout.

Dans le même esprit, chez Mazzieri, l'adjoint du maire, Marceau, s'effondre de culpabilité après avoir jeté l'idiot dans le puits. Comme pour s'en libérer, il se rend chez le curé pour faire état de la situation: il déclare être entré chez madame Henri, la mère de l'idiot, et l'avoir trouvée avec un fusil à côté d'elle. «Je me suis dit qu'elle avait fini par s'en débarrasser. Je suis parti.» $(D T, 50)$, ment-il. Cette accusation gratuite, alors même que tous ignorent la disparition de l'idiot, ne fait que souligner le statut de la vérité quand un seul récit agit pour guide. Plus tard, n'arrivant pas à se souvenir d'avoir fait levier avec le corps de l'idiot pour le jeter dans le puits, Marceau doutera de sa propre version: «Ce n'était peut-être pas vraiment lui après tout.» $(D T, 72)$

Mais c'est dans Le chasseur inconnu que nous rencontrons le plus explicitement cette «renarrativisation » constante des crimes. Le titre même du roman évoque la fiction du Professeur pour expliquer la mort de la coiffeuse Campeau, pourtant fusillée devant témoins. "Personne ne pratique la chasse dans ce village, et le hameau voisin est à trente kilomètres, mais allons, quelqu'un de la ville a peut-être décidé de venir taquiner le faisan dans le coin» $(C I, 145)$, ironise le boulanger pour souligner l'absurdité de la situation dans laquelle le village se trouve englué. Les deux narrations du roman permettent de voir à l'œuvre cette construction de la vérité, alors que la version officielle du lundi, avec le maire, le curé et tous les notables présents pour la subir, apparaît au lecteur lourde des manipulations du vendredi, de la vérité détournée et reconstruite: «Il nous faut définitivement incriminer ce Démon» (CI, 162), dira le Professeur à propos du Recenseur. Nous verrons aussitôt la preuve de sa culpabilité construite dans la séance paroissiale du lundi.

Le démon. Nous avons là un mot utile en l'absence d'enquête, car il désigne un mal absolu déjà narrativisé qui, précisément, se passe de justification. La cantilène implicitement attachée à la jeune fille morte chez Mazzieri en désigne l'hypothèse. Un villageois en colère écrira, dans son orthographe chancelante, "VATAN" à l'adresse de Paul Barabé, en manière de double injonction: pars, tu es Satan, vade retro satana. N'obtempérant pas, il sera tué, à l'instar du Recenseur chez Fortier et du loup chez Roy.

Uri Eisenzweig, dans son étude sur le roman policier, montre qu'en raison de son caractère singulièrement véridique, le «récit du détective exclut, au fond, toute possibilité de coexistence entre lui et le texte qui le contient. Racontant de manière radicalement différente ce qui se veut déjà raconté, le détective rompt la cohérence

21 Ces idées sont discutées chez Micheline Cambron, à partir de la réflexion de Paul Veyne. Voir Micheline Cambron, «Problèmes du récit», Une société, un récit. Discours culturel au Québec, 1967-1976, Montréal, l’Hexagone, coll. «Essais littéraires», 1989, p. 15-48. 
textuelle entière ${ }^{22} »$. Or, les trois romans étudiés ici se refusent à rompre cette cohérence. On peut même dire que l'absence de professionnels du crime sert ce dessein: montrer une communauté et ses fictions, sans véritable révélateur diégétique. Plutôt, le révélateur se fait narratif, par l'enchaînement des narrations et une place laissée aux voix les plus autoritaires de la communauté pour ajouter à la rumeur, pour grossir les faits, les détourner, les contredire. Il s'agit de maintenir l'ordre interne, car la communauté n'en connaît pas d'autres - sinon la menace externe de la nature, toujours prête à faire basculer la culture dans le chaos. Rien ni personne de l'extérieur ne convainc assez les villageois, dans les trois romans, pour éveiller leur désir de justice; on aurait pu, l'eût-on souhaité, aller chercher ailleurs la police et valoriser son expertise. Mais au contraire, l'ailleurs, c'est la menace. L'ailleurs, c'est le plus grand danger. Quant à la révélation des crimes, elle n'agit narrativement qu'envers le lecteur: elle ne touche jamais les membres de la communauté, les gardant protégés, encore une fois, de la vérité. La communauté est réduite à sa benoîte distance, soumise aux récits que nous savons faux, mais que tout un ordre et des lois donnent pour vrais.

\section{LES RETOURS À DISTANCE}

L'origine et le meurtre appartiennent à la même ligne causale, brouillée derrière le même écran dans les romans qui nous intéressent. La pureté de cette origine, en tant que gage de la continuité communautaire, est sacrifiée par un pouvoir dévoyé, maire ou aspirant, mystérieux Professeur; ce sacrifice, toutefois, en appelle un autre, qui sert à repousser l'étrangeté de ces morts. C'est en ce sens que le bouc émissaire ne saurait être, structurellement, qu'un étranger. Georg Simmel déplie cette logique en évoquant l'étranger moins comme un vagabond - qui arrive un jour et repart le lendemain - que comme celui qui arrive un jour et reste le lendemain ${ }^{23}$. Cet étranger fait advenir le lointain à proximité, il signifie la différence de ce lointain juxtaposé à l'ici. Pour inverser les constats d'Alex Gagnon, nous pourrions dire que l'étranger, parce qu'étranger, est criminel: sa communauté du dehors l'incrimine. En condamnant l'étranger, les régions représentées chez Mazzieri, Roy et Fortier répètent l'idylle de l'ici, repoussant en dehors ce qui germe à l'intérieur.

L'actualisation à distance permet ainsi aux œuvres de représenter un temps prémoderne du haut d'une modernité avancée. Comme devant le film The Village, le lecteur en vient à se demander comment les sujets des récits peuvent se contenter de ces demi-vérités, de ces mythes qui gangrènent le réel, des injustices érigées en système. Cela va sans dire, une telle construction révèle davantage de notre époque que de celle décrite: ainsi va la politique de la lecture. Voilà où il faut conclure.

Les œuvres que nous avons analysées ne se contentent pas de faire retour vers des lieux reculés, espaces régionaux souvent délaissés par le langage artistique: elles

22 Uri Eisenzweig, Le récit impossible. Forme et sens du roman policier, Paris, Christian Bourgois éditeur, 1986, p. 151.

23 On retrouve ce raisonnement dans ses «Digressions sur l'étranger» [1908], repris dans L'École de Chicago, textes traduits et présentés par Yves Grafmeyer et Isaac Joseph, Paris, Aubier-Montaigne, coll. «RES. Champ urbain», 1984 [1979], p. 53. 
présentent ces régions à l'époque de leur légitimé culturelle, alors qu'elles fondaient une morale ambitieuse, distribuée - postule-t-on - et écoutée. Ce geste actualisant rappelle à divers égards les entreprises de William Faulkner. Devant les États du Sud déchus de leur splendeur, le romancier américain raconte ce qui y était et qui n'y est plus, avec un effet de ressac pour le moins violent. L'injustice de la condamnation à la fin de Sanctuaire rappelle la conclusion de Trois fois la bête; la lutte des points de vue narratifs dans Le bruit et la fureur fait retour dans Le discours sur la tombe de l'idiot; la narration particulière du Chasseur inconnu reprend explicitement certains traits de la célèbre nouvelle «Une rose pour Emily». Il y a là, évidemment, un peu d'anecdotique. Toutefois, on doit mesurer que la région représentée depuis sa déchéance revêt dans tous les cas les mêmes artifices de mise à distance, ceux de narrations trop collées aux rumeurs pour que le lecteur en soit le dupe, toujours jugeant le procès qui se fait à l'aune de normes modernes éclairées.

Ce trait témoigne, pour beaucoup, depuis ce que l'on nomme dans le discours social «l'ère postfactuelle», d'une mise en perspective tentée par la littérature contemporaine. Alors que la judiciarisation de nos rapports sociaux et les autorités spécialisées incompréhensibles et surrationalisées ${ }^{24}$ présentent les écueils de notre modernité, les œuvres étudiées rappellent ce qu'offrait l'ordre prémoderne: des récits de croyance plutôt que de savoir, où les faits ne constituaient que des béquilles narratives pour justifier une téléologie de la continuité, du même, de l'ordre. On reprend alors le texte de la vie simple en région, sans jargon, sans complexité, mais pour en présenter la facticité. Très loin du mouvement de valorisation de la terre, libérés des ornières de la justice systématisée, comme on les retrouve dans Il pleuvait des oiseaux de Jocelyne Saucier ${ }^{25}$ ou encore Nord Alice de Marc Séguin ${ }^{26}$, les trois romans étudiés ici nous auront permis d'explorer un retour en forme de mise en garde.

24 Cette idée est au cœur des propositions de l'École de Francfort, notamment chez Theodor Adorno. On en retrouve une actualisation dans les thèses de Slavoj Zizek à propos de la «perte du Grand Autre» capable d'unir les communautés à l'ère postmoderne: «Le jargon des experts, non seulement celui des sciences naturelles mais aussi celui de l'économie et des autres sciences sociales, nous est présenté sous la forme d'un discours objectif avec lequel personne ne peut vraiment dialoguer et qui est, simultanément, intraduisible dans les termes de l'expérience commune. Pour le dire en un mot, le fossé creusé entre le discours scientifique et le sens commun est devenu infranchissable.» Slavoj Zizek, La subjectivité à venir. Essais critiques, édition établie par Frédéric Joly et François Théron, traduit de l'anglais par François Théron, Paris, Flammarion, coll. «Champs», 2006, p. 103-104.

25 Jocelyne Saucier, Il pleuvait des oiseaux, Montréal, XYZ éditeur, coll. «Romanichels», 2011, 179 p. Ce roman raconte la vie marginale de personnes âgées, retirées dans la forêt en bordure de champs de cannabis. S'ils sont donnés comme auteurs de divers forfaits, les personnages n'en sont pas moins magnifiés dans leur liberté, échappant aux serres du système.

26 Marc Séguin, Nord Alice, Montréal, Leméac, 2015, 249 p. Nous avons ici, grâce à des retours dans le temps, une valorisation d'une justice personnelle: les personnages répètent leur capacité et leur volonté de se faire justice eux-mêmes, tuant les méchants, vivant libres des complexités. On y valorise également la viande crue et la prise en charge autarcique de l'existence. 